\title{
Soft RPV through the baryon portal
}

\author{
Gordan Krnjaic ${ }^{a}$ and Yuhsin $\mathrm{Tsai}^{b}$ \\ ${ }^{a}$ Perimeter Institute for Theoretical Physics, \\ Waterloo, Ontario, Canada \\ ${ }^{b}$ Physics Department, University of California Davis, \\ Davis, California, U.S.A. \\ E-mail: gkrnjaic@perimeterinstitute.ca, yhtsai@ucdavis.edu
}

ABSTRACT: Supersymmetric (SUSY) models with $R$-parity generically predict sparticle decays with invisible neutralinos, which yield distinctive missing energy events at colliders. Since most LHC searches are designed with this expectation, the putative bounds on sparticle masses become considerably weaker if $R$-parity is violated so that squarks and gluinos decay to jets with large QCD backgrounds. Here we introduce a scenario in which baryonic $R$-parity violation (RPV) arises effectively from soft SUSY breaking interactions, but leptonic RPV remains accidentally forbidden to evade constraints from proton decay and FCNCs. The model features a global $R$-symmetry that initially forbids RPV interactions, a hidden $R$-breaking sector, and a heavy mediator that communicates this breaking to the visible sector. After $R$-symmetry breaking, the mediator is integrated out and an effective RPV $A$-term arises at tree level; RPV couplings between quarks and squarks arise only at loop level and receive additional suppression. Although this mediator must be heavy compared to soft masses, the model introduces no new hierarchy since viable RPV can arise when the mediator mass is near the SUSY breaking scale. In generic regions of parameter space, a light thermally-produced gravitino is stable and can be a viable dark matter candidate.

KeYwords: Supersymmetry Phenomenology

ARXIV EPRINT: 1304.7004 


\section{Contents}

1 Introduction 1

2 Model description $\quad 3$

2.1 Soft RPV from a broken $R$-symmetry 3

$2.2 \mathcal{B}$-term $R$-breaking 7

2.3 Spontaneous $R$-breaking $\quad 7$

3 Experimental bounds $\quad 9$

3.1 Direct production 9

$\begin{array}{ll}3.2 \text { Baryon number violation } & 10\end{array}$

$\begin{array}{lll}3.2 .1 & \text { Dinucleon decay } & 10\end{array}$

$\begin{array}{lll}3.2 .2 & n-\bar{n} \text { oscillation } & 11\end{array}$

3.2.3 Proton decay 12

$\begin{array}{lll}3.3 & \text { Displaced vertices } & 13\end{array}$

3.4 Gravitino dark matter 14

$\begin{array}{lll}4 & \text { Conclusions } & 15\end{array}$

$\begin{array}{lr}\text { A Hidden sector VEVs } & 17\end{array}$

$\begin{array}{ll}\text { B Color breaking? } & 18\end{array}$

\section{Introduction}

Weak scale supersymmetry (SUSY) has long been the leading framework for addressing the hierarchy problem. However, after accumulating over $20 \mathrm{fb}^{-1}$ of data, the LHC has yet to find any evidence of superpartners near the $\mathrm{TeV}$ scale and has already placed tight constraints on the most compelling regions of SUSY parameter space. As the lower bounds on stop and higgsino masses approach the $\mathrm{TeV}$ range, there is generic tension with naturalness; at least some fine tuning is required to stabilize the electroweak scale.

However, this interpretation of LHC results is model dependent since most SUSY searches assume $R$-parity conservation and, thus, require substantial MET in the final state. If this assumption is relaxed, sparticles can decay to standard model particles and the bounds become significantly weaker, thereby alleviating the tension with naturalness. Since none of SUSY's theoretically desirable features strictly requires $R$-parity, the current experimental situation motivates serious efforts to construct viable $R$-parity violating (RPV) alternatives. 


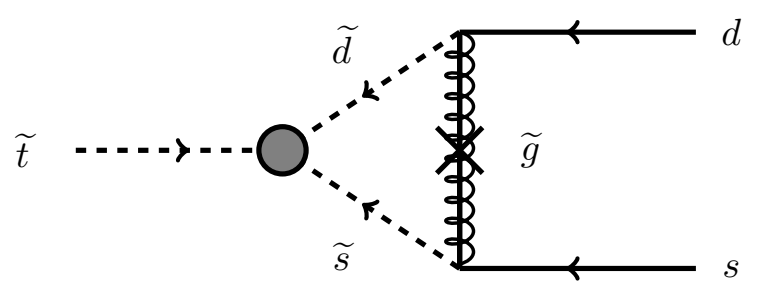

Figure 1. The loop process that yields SUSY breaking fermion-scalar RPV interactions.

In the absence of $R$-parity, the MSSM allows dangerous baryon and lepton violating operators in the superpotential

$$
W_{R P V}=\frac{\lambda_{i j k}}{2} L_{i} L_{j} \bar{E}_{k}+\lambda_{i j k}^{\prime} Q_{i} L_{j} \bar{D}_{k}+\frac{\lambda_{i j k}^{\prime \prime}}{2} \bar{U}_{i} \bar{D}_{j} \bar{D}_{k}+\mu_{L_{i}} L_{i} H_{u}
$$

and corresponding SUSY breaking terms in the soft Lagrangian

$$
\mathcal{L}_{\text {SUSY }} \supset \frac{\mathcal{A}_{i j k}}{2} \widetilde{L}_{i} \widetilde{L}_{j} \widetilde{\bar{E}}_{k}+\mathcal{A}_{i j k}^{\prime} \widetilde{Q}_{i} \widetilde{L}_{j} \widetilde{\bar{D}}_{k}+\frac{\mathcal{A}_{i j k}^{\prime \prime}}{2} \widetilde{\bar{U}}_{i} \widetilde{\bar{D}}_{j} \widetilde{\bar{D}}_{k}+\mathcal{B}_{i} \widetilde{L}_{i} H_{u}+\text { h.c. }
$$

which induce rapid proton decay and unsuppressed FCNCs if the couplings in eqs. (1.1) and (1.2) are of natural size. Since proton decay typically requires both baryon and lepton number violation, the most stringent constraints can be evaded if leptonic RPV is strongly suppressed, but baryonic RPV via $\bar{U} \bar{D} \bar{D}$ is large enough to allow the lightest squarks to decay promptly without MET $[1,2]$.

Several models in the literature satisfy these criteria. Minimal Flavor Violating (MFV) SUSY [3, 4], for example, constrains all flavor violating processes with the appropriate Yukawa couplings, which also determine the size and scope of allowed RPV interactions. However, maintaining MFV structure in a UV complete scenario requires nontrivial model building [5-7]. Similarly, "Collective RPV" [8] only allows RPV in particular combinations of couplings, so their overall effect yields the requisite suppression. Other models with similar features are found in [9-14].

Here we propose a novel scenario in which baryonic RPV arises at tree level in the soft terms, but the scalar-fermion RPV interactions in figure 1 arise only at loop level with additional suppression. These loop suppressed couplings can still be dangerous if RPV $\mathcal{A}$-terms are of order the weak scale. For instance, if the baryon number violating $\mathcal{A}$-term $\left(\mathcal{A}^{\prime \prime}\right)$ is comparable to a typical soft mass $m_{\mathcal{S}}$,

$$
\lambda^{\prime \prime} \simeq \frac{g_{s}^{2}}{16 \pi^{2}} \frac{\mathcal{A}^{\prime \prime}}{m_{\mathcal{S}}} \sim 10^{-2},
$$

this effective scalar-fermion coupling is ruled out by precision flavor constraints, which require $\lambda^{\prime \prime} \lesssim 10^{-7}$ for light flavors [15-17]. However, if these terms are generated effectively through a heavy mediator of mass $M$ that ensures $\mathcal{A}^{\prime \prime} \sim m_{\mathcal{S}}^{2} / M$, then the amount of RPV is controlled dynamically. In this framework, viable soft RPV can arise when $M$ is of order the SUSY breaking scale, so no additional hierarchy is required. Although some aspects of 
soft RPV interactions have been studied from a phenomenological perspective in [18-22], to our knowledge, a realistic model has never been realized before.

Our model features a global $R$-symmetry that forbids RPV interactions in the superpotential. This symmetry is broken in a hidden sector and communicated to the MSSM through a heavy mediator that gets integrated out to induce effective RPV $\mathcal{A}$-terms for squarks. ${ }^{1}$ If gauge mediation communicates SUSY breaking to the visible sector, the spectrum will also feature a metastable gravitino LSP that can be a viable dark matter candidate if thermally produced in the early universe.

The outline of this paper is as follows: in section 2, we list the general criteria for soft RPV and present a concrete model based on gauge mediation; in section 3 we consider the experimental constraints and map out the allowed parameter space; and in section 4 we make some concluding remarks.

\section{Model description}

On general grounds, a viable model of soft RPV requires:

- Some symmetry $G$ that forbids the usual RPV interactions in the visible sector.

- A hidden sector (generically distinct from the SUSY breaking sector) that interacts with visible fields through a heavy mediator.

- $G$-breaking triggered by soft terms in the hidden sector.

When the mediator is integrated out, the effective superpotential becomes

$$
W_{e f f} \supset \frac{X}{M} \mathcal{O}_{v i s}+X F_{G r},
$$

where $M$ is the heavy mediator mass, $X$ is a hidden sector superfield, and $F_{G}$ is a $G$ breaking spurion. The F-term for $X$ induces a $G$-breaking $\mathcal{A}$-term $\sim F_{G} / M$ for visible sector scalars, while RPV interactions involving only visible fermions are forbidden at tree level when $\langle\widetilde{X}\rangle=0$.

In this section we present a concrete model in which $G$ is an $R$-symmetry. To ensure predominantly baryonic RPV in the effective theory, we need lepton number to remain a good, accidental symmetry even after $R$-breaking. Fortunately this can be accomplished with an appropriate choice of hidden sector fields. However, SUSY breaking typically contributes an additional source of $R$-breaking, so we need to ensure that the mediation mechanism doesn't spoil the accidental lepton symmetry. Thus, we will use gauge mediation to communicate SUSY breaking to both visible and hidden sectors; perturbative gauge interactions preserve both lepton and baryon number, so leptonic RPV will not arise after $R$-breaking.

\subsection{Soft RPV from a broken $R$-symmetry}

Since $R$-symmetries are vital for generic SUSY breaking [24], we begin by imposing the following $R$-charge assignments for MSSM fields

$$
R[Q, \bar{U}, \bar{D}]=1, \quad R[L]=4 / 3, \quad R[N, \bar{E}]=2 / 3, \quad R\left[H_{u}, H_{d}\right]=0,
$$

\footnotetext{
${ }^{1} \mathrm{~A}$ global $R$-symmetry can also yield purely leptonic RPV operators [23] in the superpotential.
} 


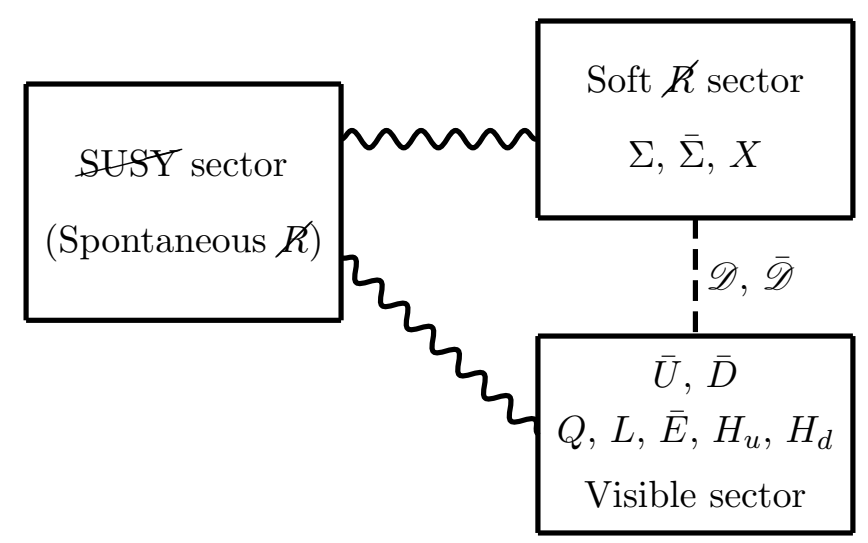

Figure 2. A schematic diagram of the relevant sectors. SUSY breaking is communicated to both the $R$-breaking and visible sectors through gauge mediation.

which forbid the RPV interactions in eq. (1.1) without imposing $R$-parity. Although this choice of $R$-charges is anomalous, heavy spectators can be added to cancel this anomaly without spoiling any of the model's features. The MSSM $\mu$ term is also forbidden at tree level, but one can arise if an additional singlet $S$ with $R$-charge +2 gets a VEV to induce $\langle S\rangle H_{u} H_{d}$ in the superpotential. It is also possible to generate weak scale higgsino and (Dirac) gaugino masses with an unbroken $R$-symmetry, though additional electroweak doublets are required $[25,26]$. Since the novel features of our model do not depend on the details of the Higgs sector, we leave this issue for future work.

The model contains three sectors depicted schematically in figure 2:

- Visible sector: contains the usual MSSM fields and interactions consistent with the $R$-symmetry, which forbids RPV.

- SUSY breaking sector: breaks both SUSY and the $R$-symmetry. SUSY breaking is mediated to the other sectors by gauge fields and decouples when all the gauge couplings vanish.

- Soft $R$-breaking hidden sector: features an additional $\mathrm{U}(1)_{H}$ gauge symmetry so hidden scalars get soft masses from gauge mediation. These soft masses can explicitly break the $R$-symmetry or induce radiative symmetry breaking through renormalization group evolution. $R$-breaking in this sector is communicated to the visible fields by heavy mediators $\mathscr{D}$ and $\overline{\mathscr{D}}$.

Even though the $R$-symmetry is also generically broken in the SUSY breaking sector, perturbative gauge interactions preserve both lepton and baryon number, so $R$-parity is not violated by gauge mediation. Visible sector RPV can only arise if the mediator connecting the visible and $R$-breaking sectors carries either lepton or baryon number. In principle, the SUSY breaking and hidden sectors may be merged, but, for simplicity of exposition we ignore this possibility here. 


\begin{tabular}{|c|cccc|}
\hline & $\mathrm{SU}(3)_{c}$ & $\mathrm{U}(1)_{Y}$ & $\mathrm{U}(1)_{H}$ & $R$ \\
\hline $\bar{U}$ & $\overline{3}$ & $-2 / 3$ & 0 & 1 \\
$\bar{D}$ & $\overline{3}$ & $1 / 3$ & 0 & 1 \\
\hline $\bar{D}$ & $\overline{3}$ & $1 / 3$ & 0 & 0 \\
$\mathscr{D}$ & 3 & $-1 / 3$ & 0 & 2 \\
\hline$X$ & 1 & 0 & 0 & -1 \\
$\Sigma$ & 1 & 0 & 1 & $3 / 2$ \\
$\bar{\Sigma}$ & 1 & 0 & -1 & $3 / 2$ \\
\hline
\end{tabular}

Figure 3. The charge assignments in our model. From top to bottom: the right-handed quarks in the visible sector, the heavy mediators $\mathscr{D} \overline{\mathscr{D}}$, the singlet $X$ connects the mediators to the $\Sigma$ fields, which are charged under the gauged $\mathrm{U}(1)_{H}$. The rightmost column lists $R$-charge assignments.

For the field content and charge assignments in table 3, the most general, renormalizable superpotential for the new states is

$$
\kappa_{i j} \epsilon^{a b c} \bar{U}_{a}^{i} \bar{D}_{b}^{j} \overline{\mathscr{D}}_{c}+\kappa_{i}^{\prime} \bar{D}^{i} \mathscr{D} X+\eta \Sigma \bar{\Sigma} X+M_{\mathscr{D}} \overline{\mathscr{D}} \mathscr{D}
$$

where $a, b, c$ are color indices and $i, j$ are flavor indices. For $M_{\mathscr{D}} \gg m_{\mathcal{S}}$, the heavy mediators $\mathscr{D}$ and $\overline{\mathscr{D}}$ are integrated out and the effective superpotential becomes

$$
-\frac{\kappa_{i[j} \kappa_{k]}^{\prime}}{M_{\mathscr{D}}} \epsilon^{a b c} \bar{U}_{a}^{i} \bar{D}_{b}^{j} \bar{D}_{c}^{k} X+\eta \Sigma \bar{\Sigma} X
$$

where the $j$ and $k$ indices are antisymmetrized. If the scalar component of $X$ gets a vacuum expectation value (VEV), there will be baryonic RPV in both the soft terms and in the effective superpotential. To emphasize the novel features of this model, we assume $\langle\widetilde{X}\rangle=0$ without essential loss of generality; we revisit this assumption in section 2.3. The effective scalar potential now contains

$$
\left|F_{X}\right|^{2} \supset-\frac{\kappa_{i[j} \kappa_{k]}^{\prime} \eta^{*}}{M_{\mathscr{D}}}(\widetilde{\Sigma} \widetilde{\bar{\Sigma}})^{*} \widetilde{\bar{U}^{i}} \widetilde{\bar{D}^{j}} \widetilde{\bar{D}^{k}}+\text { c.c. }
$$

and baryonic RPV arises from a $\widetilde{\Sigma}$ and $\widetilde{\bar{\Sigma}}$ loop with a $\mathcal{B}$-term $\left(\mathcal{B}_{\Sigma}\right)$ insertion in figure $4($ a $)$ or from $\Sigma$ and $\bar{\Sigma}$ VEVs $\left(v_{\Sigma}\right)$, which generate the diagram in figure 4(b). Note that the $R$ charges in eq. (2.2) are chosen to forbid the baryon and lepton number violating interaction $Q L \overline{\mathscr{D}}$, which generates $Q L \bar{D}$ when the mediator is integrated out. Lepton number remains a good accidental symmetry after $R$ breaking, so the only $B$ violation comes from $\bar{U} \bar{D} \bar{D}$ and higher order terms of the form $(\bar{U} \bar{D} \bar{D})^{n}$ with the same gauge-index contractions. ${ }^{2}$ Since

\footnotetext{
${ }^{2}$ Since gravity violates all global and discrete symmetries, Planck suppressed operators - e.g. $\frac{1}{M_{p l}} Q Q Q L$ and $\frac{1}{M_{p l}} \bar{U} \bar{U} \bar{D} \bar{E}$ - can still be dangerous if their coefficients are not suppressed [7]. As in the $R$-parity conserving MSSM, we assume these to be negligible or absent in a full theory valid at the Planck scale.
} 

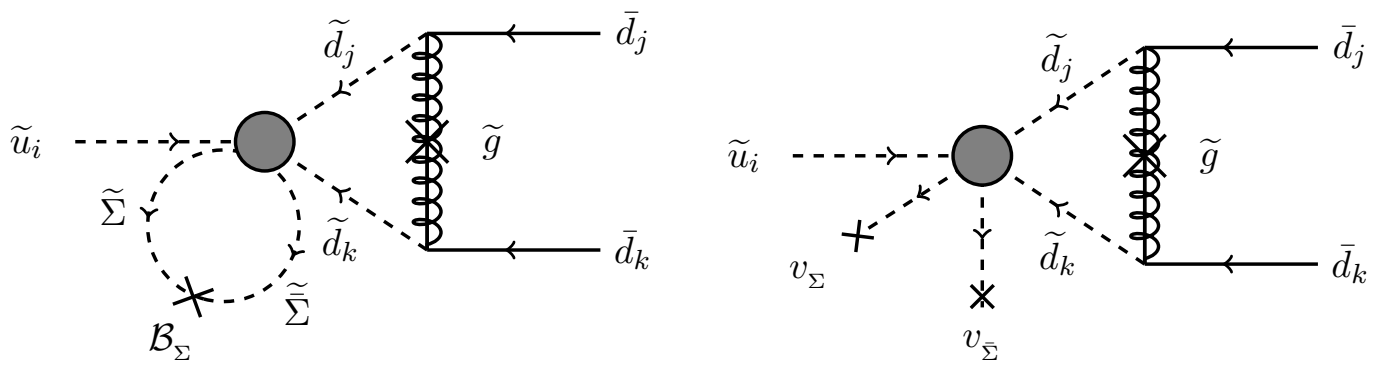

Figure 4. Effective $\lambda^{\prime \prime}$ couplings from a nonzero $\mathcal{B}$ term (a) and from spontaneous $R$-breaking (b). Diagrams with electroweak gauginos in place of gluinos also give subdominant contributions to this process.

\section{$(\mathrm{TeV})$}

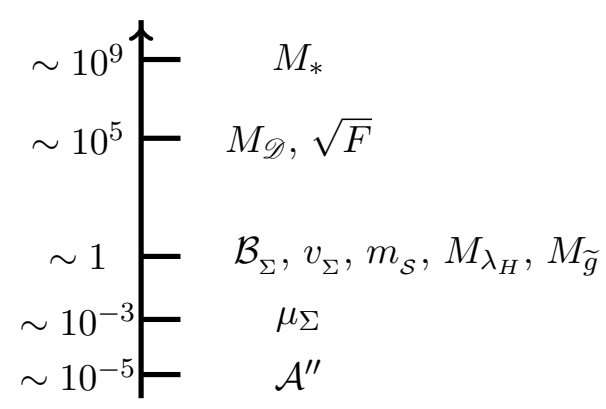

Figure 5. The hierarchies of scales in our model. Since $\mathcal{A}^{\prime \prime} \sim \mathcal{B}_{\Sigma} / M_{\mathscr{D}} \sim v_{\Sigma}^{2} / M_{\mathscr{D}}$ and $M_{\mathscr{D}} \sim \sqrt{F}$, this setup introduces no energy scales beyond those already required in conventional SUSY models.

these higher order terms induce the same $B$ violating phenomena as the leading-order $\bar{U} \bar{D} \bar{D}$ interaction, we will ignore them in our analysis of experimental constraints.

The $R$-charge assignments eq. (2.2) allow the $H_{u} L N$ operator, but forbid the Majorana term $M N N$, so Dirac neutrino masses can be generated without violating lepton number. If, instead, $L, \bar{E}$ and $N$ were all assigned unit $R$-charges, an additional discrete or flavor symmetry would be necessary to forbid lepton violation in the IR. Examples of such constructions can be found in [4-6] and [27].

Since gauge mediation communicates SUSY breaking to both visible and hidden sectors, the essential features of this model are insensitive to the details of SUSY breaking and the field content of the messenger sector. These details will, however, determine the relative sizes of $\mathcal{B}_{\Sigma}$ and $v_{\Sigma}$, so for the remainder of this paper we will remain agnostic about which diagram in figure 4 dominates and consider only the limiting cases in which only $\mathcal{B}_{\Sigma}$ or $v_{\Sigma}$ is nonzero. The general case with both contributions merely interpolates between these extremes, so our approach loses no essential generality. The ladder of scales in figure 5 summarizes the relative sizes of various inputs in our model for the convenience of the later discussion. 


\section{$2.2 \quad \mathcal{B}$-term $R$-breaking}

As a warmup to see the essential features of the model, we first consider a toy situation in which all $R$-breaking arises from a nonzero $\Sigma \bar{\Sigma} \mathcal{B}$-term, but the $\mathrm{U}(1)_{H}$ remains unbroken. The $\widetilde{\Sigma}$ and $\widetilde{\bar{\Sigma}}$ scalars get positive soft masses $\left(m_{\Sigma}\right)$ from gauge mediation so $v_{\Sigma}=v_{\bar{\Sigma}}=0$, visible sector RPV arises from the effective $\mathcal{A}$ term

$$
\mathcal{A}_{i j k}^{\prime \prime} \simeq \frac{\kappa_{i[j} \kappa_{k]}^{\prime} \eta^{*}}{16 \pi^{2}} \frac{\mathcal{B}_{\Sigma}}{M_{\mathscr{D}}} \log \frac{M_{*}^{2}}{m_{\Sigma}^{2}}
$$

where $M_{*}$ is the messenger scale, so the diagram in figure 4(a) yields

$$
\lambda_{i j k}^{\prime \prime} \simeq \frac{\kappa_{i[j} \kappa_{k]}^{\prime} \eta^{*} g_{s}^{2} \mathcal{B}_{\Sigma}}{\left(16 \pi^{2}\right)^{2} M_{\mathscr{D}} M_{\widetilde{g}}} \log \frac{M_{*}^{2}}{m_{\Sigma}^{2}}
$$

Although there are flavor-specific bounds on $\lambda^{\prime \prime}$, in principle, the index structure in $\kappa_{i[j} \kappa_{k]}^{\prime}$ can be flavor anarchic. In section 3 , we will see that, for order one $\kappa_{i j}, \kappa_{k}^{\prime}$, and $\eta$ with benchmark inputs $M_{\mathscr{D}} \sim 10^{4} \mathrm{TeV}, M_{*} \sim 10^{9} \mathrm{TeV}, \sqrt{\mathcal{B}_{\Sigma}} \sim M_{\widetilde{g}} \sim m_{\Sigma} \sim 1 \mathrm{TeV}$, the baryonic RPV coupling $\lambda^{\prime \prime}$ is naturally of order $10^{-7}$ and safe from experimental constraints; additional flavor structure in eq. (2.7) enlarges the viable parameter space, but is not an essential feature of this mechanism and lies beyond the scope of this work.

While fermion mass terms for $\Sigma, \bar{\Sigma}$ and $X$ are forbidden at tree level, a Dirac mass $\mu_{\Sigma}$ arises from hidden gaugino $\left(\lambda_{H}\right)$ interactions at one loop in figure 6 ,

$$
\mu_{\Sigma}=\frac{g_{H}^{2} \mathcal{B}_{\Sigma} M_{\lambda_{H}}}{16 \pi^{2}\left(M_{\lambda_{H}}^{2}-m_{\Sigma}^{2}\right)^{2}}\left(M_{\lambda_{H}}^{2}-m_{\Sigma}^{2}+M_{\lambda_{H}}^{2} \log \frac{m_{\Sigma}^{2}}{M_{\lambda_{H}}^{2}}\right) \sim \frac{g_{H}^{2}}{16 \pi^{2}} \frac{\mathcal{B}_{\Sigma}}{M_{\lambda_{H}}},
$$

where $M_{\lambda_{H}}$ is the hidden gaugino mass and the last expression is true in the $M_{\lambda_{H}} \sim$ $m_{\Sigma}$ regime. Although the SUSY breaking parameter $M_{\lambda_{H}}$ appears in the denominator of eq. (2.7), this diagram vanishes in the limit where SUSY is restored. An $X$ fermion mass $\sim \mu_{\Sigma} / 16 \pi^{2}$ also arises with additional loop suppression from a similar diagram with $\Sigma, \bar{\Sigma} \rightarrow X, \lambda_{H} \rightarrow \Sigma$ and $M_{\lambda_{H}} \rightarrow \mu_{\Sigma}$. In this phase, the dark gauge symmetry is unbroken, so the stable $\Sigma$ fermions annihilate to dark radiation in the early universe. The $X$ fermions decay promptly through the $X \bar{U} \bar{D} \bar{D}$ operator so long as they are heavier than the proton. If they are lighter than the $\sim 10 \mathrm{MeV}$ gravitino dark matter candidate (see section 3.4), they can contribute to the dark matter abundance without overclosing the universe.

For $v_{\Sigma}=0$ in this minimal setup, the $X$ scalar is massless at tree level and acquires a tachyonic mass from loops of $\Sigma$ and $\bar{\Sigma}$ fermions. The resulting VEV generates potentially large superpotential RPV via $\langle X\rangle \bar{U} \bar{D} \bar{D}$, so this toy scenario is unstable unless $\widetilde{X}$ acquires mass by other means. Additional mass terms for $X$ can arise either in the superpotential with additional $R$-charged fields or after SUSY breaking if the mediation mechanism gives gauge-singlets soft masses.

\subsection{Spontaneous $R$-breaking}

Now we present a more concrete scenario that generates soft RPV and solves this problem with nonzero VEVs $v_{\Sigma, \bar{\Sigma}}$ that break both $\mathrm{U}(1)_{H}$ and the $R$-symmetry. For simplicity 


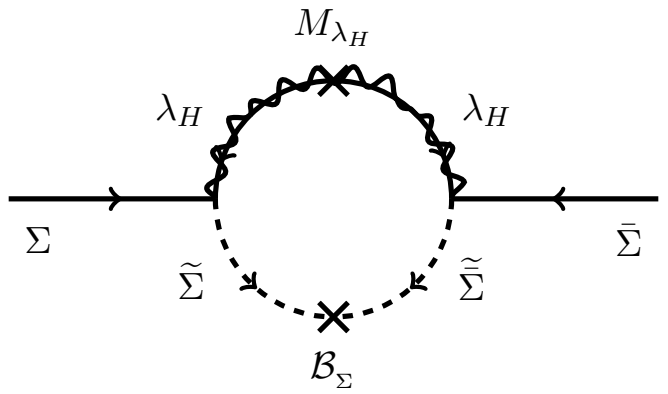

Figure 6. Dirac mass $\mu_{\Sigma}$ for $\Sigma$ and $\bar{\Sigma}$ from a nonzero $\mathcal{B}_{\Sigma}$ term.

we assume all hidden sector $\mathcal{A}$ and $\mathcal{B}$ terms vanish and set $m_{\Sigma}=m_{\bar{\Sigma}}$, so the scalar potential contains

$$
\frac{g_{H}^{2}}{2}\left(|\widetilde{\Sigma}|^{2}-|\widetilde{\bar{\Sigma}}|^{2}\right)^{2}+\eta^{2}\left(|\widetilde{\Sigma} \widetilde{\bar{\Sigma}}|^{2}+|\widetilde{X} \widetilde{\Sigma}|^{2}+|\widetilde{X} \widetilde{\bar{\Sigma}}|^{2}\right)-m_{\Sigma}^{2}\left(|\widetilde{\Sigma}|^{2}+|\widetilde{\bar{\Sigma}}|^{2}\right)
$$

where the negative mass squared can arise through RG evolution if $\Sigma$ and $\bar{\Sigma}$ couple to other fields with nonzero soft masses - see appendix A for a concrete example.

For $g_{H}^{2}>\eta^{2} / 2$, the classical minimum is

$$
v_{\Sigma}=v_{\bar{\Sigma}}=m_{\Sigma} / \eta \quad, \quad\langle\widetilde{X}\rangle=0
$$

but, quantum corrections still generate an $\widetilde{X}$ VEV. However, unlike in section 2.2, $\widetilde{X}$ now has a tree level mass of $m_{X} \sim v_{\Sigma}$, so minimizing the Coleman-Weinberg potential yields $\langle\widetilde{X}\rangle \propto \mu_{\Sigma}^{3} / m_{X}^{2}$, where

$$
\mu_{\Sigma}=\frac{g_{H}^{2} \eta^{2} v_{\Sigma}^{2} M_{\lambda_{H}}}{16 \pi^{2}\left(M_{\lambda_{H}}^{2}-m_{\Sigma}^{2}\right)^{2}}\left(M_{\lambda_{H}}^{2}-m_{\Sigma}^{2}+M_{\lambda_{H}}^{2} \log \frac{m_{\Sigma}^{2}}{M_{\lambda_{H}}^{2}}\right) \sim \frac{g_{H}^{2}}{16 \pi^{2}} \frac{\eta^{2} v_{\Sigma}^{2}}{M_{\lambda_{H}}},
$$

is the $\Sigma \bar{\Sigma}$ Dirac mass that arises from the loop-diagram in figure 7. As in eq. (2.8), we work in the $M_{\lambda_{H}} \sim m_{\Sigma}$ limit and there is a SUSY breaking mass in the denominator. However, the diagram that generates $\mu_{\Sigma}$ vanishes when SUSY is restored since $M_{\Lambda} \rightarrow 0$ and $v_{\Sigma} \rightarrow 0$ as the latter requires $m_{\Sigma}>0$ in eq. (2.9), which fails in this limit. Thus, the $\langle X\rangle \bar{U} \bar{D} \bar{D} / M_{\mathscr{D}}$ correction to fermionic RPV is subdominant to the soft contribution in figure $4(\mathrm{~b})$ for which

$$
\lambda_{i j k}^{\prime \prime}=\frac{\kappa_{i[j} \kappa_{k]}^{\prime} \eta^{*} g_{s}^{2} v_{\Sigma}^{2}}{32 \pi^{2} M_{\mathscr{D}} M_{\widetilde{g}}}
$$

Since the effective potential also contains

$$
\left|F_{X}\right|^{2}=\left|\eta \widetilde{\Sigma} \widetilde{\bar{\Sigma}}-\frac{\kappa \kappa^{\prime}}{M_{\mathscr{D}}} \widetilde{\bar{U}} \widetilde{\bar{D}} \widetilde{\bar{D}}\right|^{2}
$$

a nonzero $v_{\Sigma}$ can, in principe, trigger color breaking, however, in appendix $\mathrm{B}$ we find that color remains unbroken so long as $v_{\Sigma} \lesssim M_{\mathscr{D}}\left(m_{\widetilde{q}} / M_{\mathscr{D}}\right)^{3 / 4}$, where $m_{\widetilde{q}}$ is a typical squark mass of order the weak scale. 


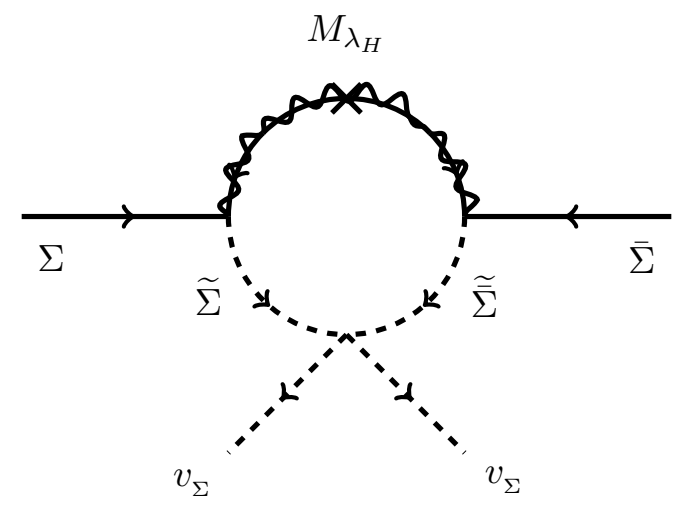

Figure 7. Loop level Dirac mass $\mu_{\Sigma}$ for $\Sigma$ and $\bar{\Sigma}$ for $v_{\Sigma} \neq 0$.

The $R$-symmetry forbids superpotential mass terms for $X, \Sigma$ and $\bar{\Sigma}$, so the hidden sector spectrum is entirely determined by SUSY breaking parameters. As in section 2.2, $\widetilde{\Sigma}$ and $\widetilde{\bar{\Sigma}}$ get gauge mediated soft masses and $\widetilde{X}$ gets a soft mass at one loop. After symmetry breaking, the $X, \Sigma, \bar{\Sigma}$, and $\lambda_{H}$ fermions mix and the resulting mass eigenstates are of order the electroweak scale. For generic mixing angles, all hidden sector mass eigenstates will be linear combinations of all four interaction eigenstates, so they all decay promptly through the $X \bar{U} \bar{D} \bar{D}$ portal.

A spontaneously broken $R$-symmetry gives rise to a massless $R$-axion that can accelerate supernova cooling and cause cosmological problems [28]. Conventionally, $R$-breaking arises only in the SUSY breaking sector and the BPR mechanism [29] generates an $R$-axion mass from a constant term in the superpotential introduced to cancel the cosmological constant. In this scenario, our hidden sector also contributes to $R$-breaking, so the physical $R$-axion is now a linear combination of SUSY breaking and hidden sector states, but still acquires a BPR mass, so we will not consider it further. Although the BPR term explicitly breaks the $R$-symmetry, we assume its existence has no additional bearing on the symmetries of our superpotential; it serves merely as a placeholder for the cosmological constant problem, which is beyond the scope of this work.

\section{Experimental bounds}

In this section we consider the experimental constraints on our realization of soft RPV. For simplicity, we will follow the organization of section 2.1 and separately constrain the cases in which the B-term and $\Sigma, \bar{\Sigma}$ VEVs are solely responsible for $R$-breaking; the most general case interpolates between these extremes. The plots in figure 8 carve out the allowed parameter space in both $\mathcal{B}$-term and spontaneously broken scenarios.

\subsection{Direct production}

Although the parameter space for RPV spectra with sparticles below a TeV has recently been reduced, the sensitivity of these bounds is driven primarily by lepton number violating processes. For purely baryonic RPV, the bounds are considerably weaker and can accommodate natural stops with $\sim 100 \mathrm{GeV}$ masses, provided they decay predominantly to dijets 

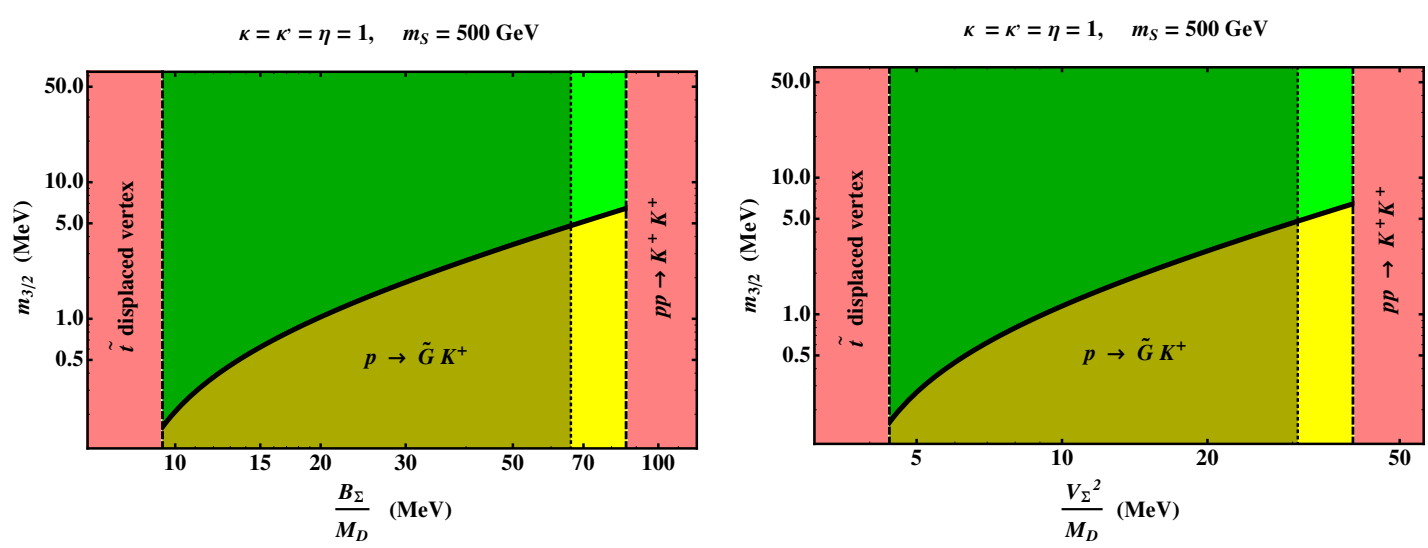

Figure 8. The parameter space for our model in the $\mathcal{B}$-term scenario (left) and in the spontaneously broken phase (right). In each case, the light (dark) green represents the allowed region where the stop decays with vertices smaller than $2 \mathrm{~mm}(10 \mathrm{~cm})$. Here we assume the most conservative scenario with $\left|\kappa_{i j}\right|=\left|\kappa_{i}^{\prime}\right|=|\eta|=1$ for all coefficients. The rates that determine the red excluded regions are quadratically sensitive to these parameters, so if light flavors have smaller coefficients, the parameter space expands considerably.

via $\bar{U} \bar{D} \bar{D}[1]$. For RPV gluinos decaying exclusively to $\widetilde{g} \rightarrow t \widetilde{t}$, the strongest experimental bound is now $\gtrsim 670 \mathrm{GeV}$ [30-35], however, recasting $R$-parity conserving SUSY searches may place a stronger $\sim 800 \mathrm{GeV}$ bound on the gluino mass [36].

\subsection{Baryon number violation}

The $\bar{U} \bar{D} \bar{D}$ interaction explicitly violates baryon number, so our model faces constraints from the null results of several low energy searches. The strongest limits come from the bounds on the characteristic timescales for dinucleon decay $\left(p p \rightarrow K^{+} K^{+}\right)[37]$ and neutron-antineutron oscillation $(n-\bar{n})[38]$

$$
\tau_{p p \rightarrow K K} \geq 1.7 \times 10^{32} \text { yrs. }, \quad \tau_{n-\bar{n}} \geq 2.44 \times 10^{8} \text { sec. },
$$

and from proton decay via $p \rightarrow K^{+} \nu$, for which the bound is [39]

$$
\tau_{p \rightarrow K^{+} \nu} \geq 2.3 \times 10^{33} \mathrm{yrs} .
$$

Although our model doesn't violate lepton number, this bound conservatively constrains the $p \rightarrow K^{+} \widetilde{G}$ decay, which has similar kinematics for a sufficiently light gravitino.

\subsubsection{Dinucleon decay}

Following Goity and Sher [16], the dinucleon decay rate for the dominant processes shown in figure 9 is

$$
\Gamma_{p p \rightarrow K K} \sim \rho_{N} \frac{128 \pi \alpha_{s}^{2} \Lambda^{10}}{m_{p}^{2} m_{\widetilde{u}}^{8} M_{\widetilde{g}}^{2}}\left(\lambda_{u d s}^{\prime \prime}\right)^{2},
$$

where $m_{\widetilde{u}}$ is the lightest up-type squark mass, $\rho_{N} \sim 0.25 / \mathrm{fm}^{3}$ is the density of nuclear matter and $\Lambda$ is the characteristic hadronic energy-scale. Here we assume $M_{\widetilde{C}}>M_{\widetilde{g}} \alpha / \alpha_{s} \gtrsim$ 

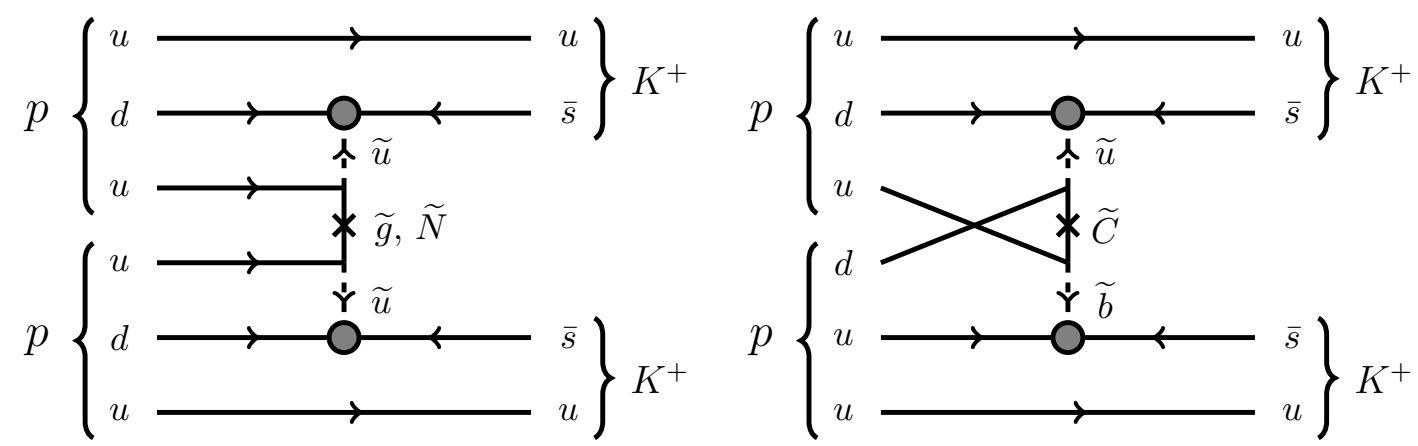

Figure 9. Dinucleon decay via baryonic RPV interactions. In the text we assume gluino exchange (left) dominates.

$220 \mathrm{GeV}$, so the gluino exchange diagram in figure 9 dominates. Thus, satisfying the experimental bound $\tau_{p p \rightarrow K K} \geq 1.7 \times 10^{32}$ yrs. requires

$$
\lambda_{u d s}^{\prime \prime} \lesssim 2.5 \times 10^{-7}\left(\frac{150 \mathrm{MeV}}{\Lambda}\right)^{5 / 2}\left(\frac{M_{\widetilde{g}}}{800 \mathrm{GeV}}\right)^{1 / 2}\left(\frac{m_{\widetilde{u}}}{500 \mathrm{GeV}}\right)^{2}
$$

Translating this into a constraint on the $\mathcal{B}$-term scenario $\left(v_{\Sigma}=0\right)$ in section 2.2 , we have

$$
\frac{\mathcal{B}_{\Sigma}}{M_{\mathscr{D}}} \lesssim 81 \mathrm{MeV}\left(\frac{150 \mathrm{MeV}}{\Lambda}\right)^{5 / 2}\left(\frac{M_{\widetilde{g}}}{800 \mathrm{GeV}}\right)^{1 / 2}\left(\frac{m_{\widetilde{u}}}{500 \mathrm{GeV}}\right)^{2}\left(\eta^{*} \kappa_{u[d} \kappa_{s]}^{\prime}\right)^{-1}
$$

where we have set $M_{*}=10^{9} \mathrm{GeV}$ and $m_{\Sigma}=500 \mathrm{GeV}$ inside the log of eq. (2.7). Similarly, for the spontaneous $R$-breaking scenario $\left(\mathcal{B}_{\Sigma}=0\right)$ in section 2.3 , the corresponding bound is extracted from eq. (2.12)

$$
\frac{v_{\Sigma}^{2}}{M_{\mathscr{D}}} \lesssim 42 \mathrm{MeV}\left(\frac{150 \mathrm{MeV}}{\Lambda}\right)^{5 / 2}\left(\frac{M_{\widetilde{g}}}{800 \mathrm{GeV}}\right)^{1 / 2}\left(\frac{m_{\widetilde{u}}}{500 \mathrm{GeV}}\right)^{2}\left(\eta^{*} \kappa_{u[d} \kappa_{s]}^{\prime}\right)^{-1}
$$

Unlike similar processes in MFV SUSY [4] where the light quark couplings are Yukawa suppressed, our setup imposes no necessary hierarchies in the RPV couplings.

\subsection{2 $n-\bar{n}$ oscillation}

Unlike dinucleon decay, $n-\bar{n}$ oscillation also requires flavor violation from $R$-parity conserving vertices. However, aside from the baryon violating $\mathcal{A}$-term, all visible sector soft masses arise directly from gauge mediation, so their flavor structure comes entirely from Yukawa couplings. Thus, up to an overall coefficient, our $n-\bar{n}$ oscillation amplitudes are identical to those computed in [4].

Chirality-preserving flavor-violating masses arise predominantly from MSSM $F$-terms after SUSY and electroweak symmetry breaking through

$$
\widetilde{Q}^{\dagger}\left(v_{u}^{2} Y_{u} Y_{u}^{\dagger}+v_{d}^{2} Y_{d} Y_{d}^{\dagger}\right) \widetilde{Q}
$$

and similar terms for $\widetilde{\bar{U}}$ and $\widetilde{\bar{D}}$, where $Y_{u, d}$ are Yukawa matrices. For simplicity, we take the Higgs doublet VEVs $v_{u, d}$ to be at the soft scale $\sim m_{\mathcal{S}}$. In gauge mediation, chirality flipping 


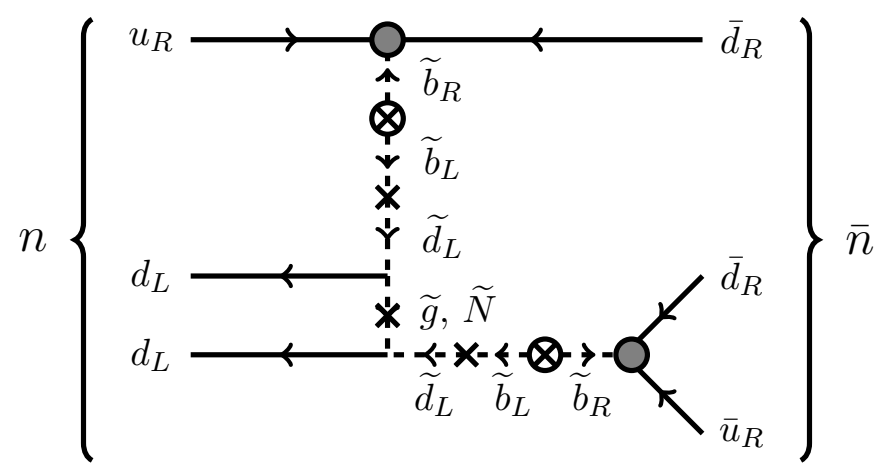

Figure 10. The dominant diagram for neutron anti-neutron oscillation. All $R$-parity conserving soft masses and $\mathcal{A}$-terms are consistent with MFV.

$\mathcal{A}$-terms arise only at higher order and suffer both Yukawa and loop suppression, so they are typically smaller than soft masses. However, different realizations of gauge mediation give rise to $\mathcal{A}$ terms with different degrees of suppression relative to the soft scale. Since we remain agnostic about the details of the messenger sector, we conservatively parametrize any possible suppression with the general ansatz $\mathcal{A} \equiv \epsilon m_{\mathcal{S}}$.

Putting all the squarks at a common soft mass $m_{\widetilde{q}} \sim m_{\mathcal{S}}$, the amplitude for the dominant diagram shown in figure 10 is

$$
\mathcal{M}_{n-\bar{n}} \sim g_{s}^{2} \epsilon^{2} \lambda^{6} \Lambda\left(\frac{\Lambda}{m_{\widetilde{q}}}\right)^{4}\left(\frac{\Lambda}{M_{\widetilde{g}}}\right)\left(\lambda_{u d b}^{\prime \prime}\right)^{2}
$$

where $\lambda \simeq 0.23$ comes from the approximate CKM matrix parametrization in [4]. The oscillation timescale is approximately $\tau_{n-\bar{n}} \sim \mathcal{M}^{-1}$, thus the experimental bound $\tau_{n-\bar{n}} \geq$ $2.44 \times 10^{8}$ sec. requires

$$
\lambda_{u d b}^{\prime \prime} \lesssim 1.7 \times 10^{-6} \epsilon^{-2}\left(\frac{m_{\widetilde{q}}}{500 \mathrm{GeV}}\right)^{4}\left(\frac{250 \mathrm{MeV}}{\Lambda}\right)^{6}\left(\frac{M_{\widetilde{g}}}{800 \mathrm{GeV}}\right)
$$

which is weaker than the bound from dinucleon decay in eq. (3.4) even when $\epsilon$ is order one.

\subsubsection{Proton decay}

Since gauge-mediation typically features a light, sub-GeV gravitino, proton decay to $K^{+} \widetilde{G}$ through the diagram in figure 11 may be kinematically allowed. The rate for this process is

$$
\Gamma_{p \rightarrow K^{+} \widetilde{G}} \sim \frac{m_{p}}{8 \pi}\left(\frac{\Lambda}{m_{\widetilde{u}}}\right)^{4}\left(\frac{\Lambda^{2}}{\sqrt{3} m_{3 / 2} M_{p l}}\right)^{2}\left(\lambda_{u d s}^{\prime \prime}\right)^{2}
$$

and the lifetime for this channel must be longer than $2.3 \times 10^{33}$ yrs., so the gravitino mass bound is

$$
m_{3 / 2} \geq 4.7 \mathrm{MeV}\left(\frac{\Lambda}{250 \mathrm{MeV}}\right)^{4}\left(\frac{500 \mathrm{GeV}}{m_{\widetilde{u}}}\right)^{2}\left(\frac{\lambda_{u d s}^{\prime \prime}}{10^{-7}}\right)
$$


For $m_{3 / 2} \gtrsim 5 \mathrm{MeV}$, this implies a lower bound on the SUSY breaking scale

$$
\sqrt{F} \gtrsim 3.2 \times 10^{5} \mathrm{TeV}
$$

If minimal gauge mediation gives rise to soft masses, the messenger scale $M_{*}$ must also satisfy

$$
M_{*} \gtrsim 1.3 \times 10^{9} \mathrm{TeV}\left(\frac{500 \mathrm{GeV}}{m_{\mathcal{S}}}\right) .
$$

\subsection{Displaced vertices}

To avoid MET searches at the LHC, sparticles must decay on collider timescales, so there is an upper bound on the lightest squark's lifetime. Although there are many LHC searches for displaced vertices [30, 40], hadronically-decaying long-lived particles are significantly harder to constrain [41]; viable decay lengths can even exceed $\sim 10 \mathrm{~cm}$, so a dedicated search is necessary. Given these uncertainties, we consider the experimental bounds in two regimes: for prompt decays, we conservatively require decay lengths $\ell_{\widetilde{q}}<2 \mathrm{~mm}$; for signatures with viable displaced vertices, we demand $\ell_{\widetilde{q}}<10 \mathrm{~cm}$, so most sparticles decay inside the tracker before reaching the hadronic calorimeter (HCAL), but may still be found with a dedicated search.

The width for a hardronically decaying stop $\mathrm{NLSP}^{3}$ in its rest frame is

$$
\Gamma_{\widetilde{t} \rightarrow \bar{q} \bar{q}}=\frac{m_{\widetilde{t}}}{8 \pi} \sin ^{2} \theta_{\widetilde{t}}\left|\lambda_{t q q}^{\prime \prime}\right|^{2},
$$

where $\theta_{\widetilde{t}}$ is the stop mixing angle. In the lab frame, the decay length is $\ell_{\widetilde{t}} \simeq \gamma \Gamma_{\widetilde{t} \rightarrow q \bar{q}}^{-1}$, where $\gamma$ is the stop boost factor; for a $300 \mathrm{GeV}$ stop and an $800 \mathrm{GeV}$ gluino produced at rest, $\gamma \sim 2$. For the remainder of this section we assume, for simplicity, that $\gamma \sin ^{2} \theta_{\tilde{t}}=1$.

Assuming the dominant stop decay is $\widetilde{t} \rightarrow \bar{d} \bar{s}$, the bound on $\lambda_{t d s}^{\prime \prime}$ is

$$
\lambda_{t d s}^{\prime \prime}>(0.26-1.8) \times 10^{-7}\left(\frac{300 \mathrm{GeV}}{m_{\tilde{t}}}\right)^{1 / 2} .
$$

where the left and right numbers represent the bound assuming $10 \mathrm{~cm}$ and $2 \mathrm{~mm}$ displacedvertex limits, respectively. For the $\mathcal{B}$-term scenario $\left(v_{\Sigma}=0, \mathcal{B}_{\Sigma} \neq 0\right)$ in section 2.2 , this implies

$$
\frac{\mathcal{B}_{\Sigma}}{M_{\mathscr{D}}} \gtrsim(8.3-58) \times \mathrm{MeV}\left(\frac{m_{\widetilde{g}}}{800 \mathrm{GeV}}\right)\left(\frac{300 \mathrm{GeV}}{m_{\tilde{t}}}\right)^{1 / 2}\left(\kappa_{t[d} \kappa_{s]}^{\prime} \eta^{*}\right)^{-1}
$$

with $m_{\Sigma}=1 \mathrm{TeV}$ and $M_{*} \sim 10^{9} \mathrm{TeV}$ inside the log in eq. (2.7). Similarly, for the spontaneously broken scenario $\left(v_{\Sigma} \neq 0, \mathcal{B}_{\Sigma}=0\right)$ in section 2.3 , we have

$$
\frac{v_{\Sigma}^{2}}{M_{\mathscr{D}}} \gtrsim(4.3-31) \times \mathrm{MeV}\left(\frac{m_{\widetilde{g}}}{800 \mathrm{GeV}}\right)\left(\frac{300 \mathrm{GeV}}{m_{\widetilde{t}}}\right)^{1 / 2}\left(\kappa_{t[d} \kappa_{s]}^{\prime} \eta^{*}\right)^{-1}
$$

\footnotetext{
${ }^{3}$ For typical SUSY breaking scales we consider, the gravitino is the LSP, though for extremely high SUSY breaking scales, this need not be the case.
} 


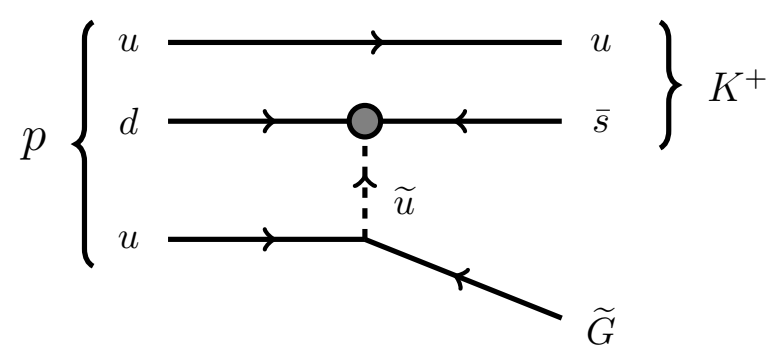

Figure 11. Proton decay via $p \rightarrow K^{+} \widetilde{G}$.

These bounds assume the stop is the lightest squark and decays predominantly through RPV interactions. Thus, the only other kinematically allowed process $\widetilde{t} \rightarrow t \widetilde{G}$ must have a negligible branching ratio, which requires

$$
\Gamma_{\widetilde{t} \rightarrow \bar{q} \bar{q}} \gg \Gamma_{t \widetilde{G}}=\frac{m_{\widetilde{t}}^{5}}{16 \pi F^{2}}
$$

As long as the SUSY breaking scale satisfies $\sqrt{F}>10^{2} \mathrm{TeV}$, the RPV branching ratio exceeds $99 \%$. This constraint is trivially satisfied by considerations from proton decay in section 3.2 .3 above.

\subsection{Gravitino dark matter}

Since gauge mediation communicates SUSY breaking to the visible sector, the gravitino is the LSP with mass $m_{3 / 2} \sim F / M_{p l} \sim \mathcal{O}(10) \mathrm{MeV}$ for $\sqrt{F} \sim 10^{8} \mathrm{GeV}$. In this mass range $m_{3 / 2}<m_{p}$, so the process $\widetilde{G} \rightarrow q q q$ is kinematically forbidden and the gravitino is stable. Since sparticles rarely decay to gravitinos and their annihilation rate is suppressed by the SUSY breaking scale, the present day abundance is thermally generated [13]

$$
\Omega_{3 / 2} h^{2} \simeq 0.1\left(\frac{T_{R}}{10^{5} \mathrm{GeV}}\right)\left(\frac{m_{3 / 2}}{20 \mathrm{MeV}}\right)^{-1}\left(\frac{M_{\widetilde{g}}}{800 \mathrm{GeV}}\right)^{2}
$$

where $T_{R}$ is the reheating temperature, so the RPV gravitino is a viable dark matter candidate. Although the scenario in [13] assumes $R$-parity conservation, the analysis is applicable so long as thermal gravitino production in the early universe is not upset by additional decays or annihilation processes.

Thermal gravitino dark matter can also arise in models of spontaneous blilnear RPV [42-44]. In that scenario, the gravitino is metastable on cosmological timescales, but can decay leptonically at late times and be observed through indirect detection. Our basic model features a stable gravitino and only generates baryonic RPV; lepton number remains a good accidental symmetry, so this potential signature is absent. However, in the presence of additional $R$-breaking states or an alternative SUSY breaking mediation mechanism, it is possible to generate small leptonic RPV to allow gravitino decay, but this is beyond the scope of the present work. 


\section{Conclusions}

In this paper we have presented a new realization of weak scale SUSY with $R$-parity violation. Unlike conventional scenarios, suppressed baryonic RPV arises in the soft terms when an $R$-symmetry is broken in a hidden sector and a heavy mediator is integrated out; lepton number remains a good accidental symmetry. RPV interactions between quarks and squarks arise at one loop and receive additional suppression. The model features light $(\sim$ few $100 \mathrm{GeV}$ ) squarks that decay promptly to hadrons and evade LHC searches in viable regions of parameter space safe from flavor constraints. Without additional, colored field content, RG evolution from a high UV scale naturally yields comparable squark and gluino masses in the IR, so there is tension in maintaining light squarks to preserve naturalness while keeping gluinos near the $\mathrm{TeV}$ scale to evade stronger collider bounds. [45] Mild tuning or additional field content may be required for a fully natural spectrum, but a detailed analysis is beyond the scope of this work.

For weak-scale $R$-breaking, the heavy mediator masses can be near the SUSY breaking scale $\sqrt{F} \sim 10^{8} \mathrm{GeV}$ to generate RPV couplings with the requisite suppression, so the model requires no new scales beyond those already present in conventional SUSY models. If gauge mediation communicates SUSY breaking, the model also features a light $\sim 1-100 \mathrm{MeV}$ gravitino with a thermal abundance. For a reheating temperature of order $10^{5} \mathrm{GeV}$ and a weak scale gluino, a gravitino in this mass range is a viable dark matter candidate.

Like MFV SUSY [4], our soft-RPV scenario predicts hadronically decaying squarks and gluinos with high multiplicity multijet final states for all colored LHC-accessible states. Both frameworks predict predominantly baryonic RPV and can accommodate light squarks, but the flavor structure in our case is not fixed by the Yukaka and CKM matrices, so the branching ratios to different quark flavors can differ by order one amounts. Furthermore, our model is also easily falsifiable: if SUSY stabilizes the hierarchy problem and features baryonic soft-RPV, then squark or gluino resonances will be discovered at the LHC, while leptonic RPV will be absent.

Although small baryonic RPV ameliorates the tension between weak-scale sparticle masses and LHC direct production constraints, this feature, by itself, does not yield a $\sim 126 \mathrm{GeV}$ Higgs. This issue is well known and various solutions have been proposed in the literature [46-55]. Our scenario is a proof of concept to show that baryonic RPV arising from soft terms can evade production bounds; generating a viable Higgs mass in this framework is compatible with many of the known strategies. The benchmark example in this paper uses gauge mediation to generate soft masses, so stop mixing is small, but other tools including non-decoupling D-terms [50, 53], induced electroweak symmetry breaking [54], or heavy superpartners [55] can lift the Higgs mass without spoiling any of the model's essential features. For example, following [50], we can extend the gauged $\mathrm{U}(1)_{H}$ symmetry to the SM sector and include the Higgs in the D-term potential

$$
\frac{g_{H}^{2}}{2}\left(q_{H}\left|H_{u}\right|^{2}-q_{H}\left|H_{d}\right|^{2}+|\widetilde{\Sigma}|^{2}-|\widetilde{\bar{\Sigma}}|^{2}\right)^{2} .
$$


Since the $\Sigma$ VEVs and masses are at the TeV scale, we can integrate them out after $\mathrm{U}(1)_{H}$ is broken in eq. (2.11) to induce an extra contribution to the Higgs quartic coupling

$$
\frac{g_{H}^{2} q_{H}^{2}}{2}\left(\left|H_{u}\right|^{2}-\left|H_{d}\right|^{2}\right)^{2} \times\left(1+\frac{g_{H}^{2} v_{\Sigma}^{2}}{4 m_{\Sigma}^{2}}\right)^{-1} .
$$

With these corrections, the Higgs having an MSSM potential can now obtain the required mass with an order one $g_{H}$ used in appendix A. The few TeV scale $g_{H} v_{\Sigma}$ studied there also satisfy the bounds from the electroweak precision measurement and the $Z^{\prime}$ searches. $^{4}$ The bound constraints the mixing between the $\mathrm{SM}$ and $\mathrm{U}(1)_{H}$ gauge bosons. Although a detailed analysis is required, this can be a reasonable way to generate the observed Higgs mass even without a large stop A-term in a gauge mediated scenario. However, we emphasize that, for our purposes, gauge mediation is merely a convenient mechanism to generate soft masses without violating lepton or baryon number; any alternative for which this holds true is similarly viable and would be interesting to study.

If $R$-breaking arises from a $\mathcal{B}$-term for $\Sigma$ and $\bar{\Sigma}$ as in section 2.2 , the model requires either non-minimal gauge mediation to generate sizable $\mathcal{B}$-terms, or another mediation mechanism that preserves the accidental lepton symmetry. We leave these model building details for future work. For the more-concrete spontaneous $R$-breaking scenario in section 2.3, the model requires either additional fields to drive radiative symmetry breaking for $\Sigma$ and $\bar{\Sigma}$ or an alternative to gauge mediation that results in tachyonic soft masses in the hidden sector. In appendix A we show that radiative symmetry breaking is feasible, but leave other alternatives for future work.

Grand unification with RPV is challenging because both lepton and baryon number violating RPV interactions generally arise from the same interaction term. In $\mathrm{SU}(5)$, for instance, $\bar{U} \bar{D} \bar{D}, Q L \bar{D}$ and $L L \bar{E}$ all live in the same $10 \overline{5} \overline{\mathrm{UV}}$ operator, so generating predominantly baryonic RPV at low energies requires additional model building gymnastics [11]. In our case, the $R$-charge assignments differ for quark and lepton superfields, so it is not clear whether grand unification is possible.

\section{Acknowledgments}

We thank Csaba Csaki, Ben Heidenreich, Markus Luty, Surjeet Rajendran, Carlos Tamarit, John Terning and Yue Zhao for helpful conversations. Additional thanks to Ben Heidenreich, Carlos Tamarit, and John Terning for comments on the draft. Research at the Perimeter Institute is supported in part by the Government of Canada through Industry Canada, and by the Province of Ontario through the Ministry of Research and Information (MRI). YT thanks the Perimeter Institute for its hospitality while this work was in progress. YT is supported by the Department of Energy under grant DE-FG02-91ER406746.

Addendum (January 2014). Since this paper was originally submitted for publication, the LHC constraints on SUSY scenarios have improved [56]. Nearly model independent bonds on gluino masses are now in the $\sim \mathrm{TeV}$ range and squark mass-bounds have also

\footnotetext{
${ }^{4}$ See, for example, the figure 4 in [10].
} 
improved considerably. In this light, the LHC motivation for baryonic RPV is somewhat diminished, though the essential features of the soft RPV scenario remain viable even at higher sparticle mass scales.

\section{A Hidden sector VEVs}

Throughout the paper, we have assumed that the $\Sigma$ and $\bar{\Sigma}$ scalars acquire negative masssquared parameters to induce spontaneous symmetry breaking. Since the minimal superpotential only allows the $\Sigma X \bar{\Sigma}$ interaction and gauge mediation gives rise to positive soft masses, the setup requires either a nonminimal messenger sector to generate negative soft masses or substantial RG evolution. Since we are agnostic about the details of gauge mediation, here we present a concrete example of radiative $R$-breaking in the hidden sector as a proof of concept.

If the $\Sigma$ scalars also couple to chiral fields $Y$ and $\bar{Y}$ with identical $R$-charges of $1 / 4$ and $\mathrm{U}(1)_{H}$ charges of $\mp 1 / 2$, the superpotential also contains

$$
W \supset \eta \Sigma X \bar{\Sigma}+\lambda_{Y} \Sigma Y^{2}+\lambda_{\bar{Y}} \bar{\Sigma} \bar{Y}^{2}
$$

where $\eta, \lambda_{Y}$, and $\lambda_{Y}$ are order one parameters. Including $\mathrm{U}(1)_{H}$ gauge interactions, the full set of RGEs is

$$
\begin{aligned}
\frac{d g_{H}}{d t} & =\frac{5 g_{H}^{3}}{32 \pi^{2}} \\
\frac{d \lambda_{Y, \bar{Y}}}{d t} & =\frac{\lambda_{Y, \bar{Y}}}{16 \pi^{2}}\left(\frac{5}{2} \lambda_{Y, \bar{Y}}^{2}-3 g_{H}^{2}\right) \\
\frac{d \eta}{d t} & =\frac{\eta}{16 \pi^{2}}\left(3 \eta^{2}-4 g_{H}^{2}\right) \\
\frac{d m_{\Sigma}^{2}}{d t} & =\frac{1}{16 \pi^{2}}\left[\eta^{2}\left(2 m_{\Sigma}^{2}+m_{\bar{\Sigma}}^{2}+m_{X}^{2}\right)+4 \lambda_{Y}^{2}\left(m_{\Sigma}^{2}+m_{Y}^{2}\right)+\frac{g_{H}^{2}}{2}\left(-2 m_{\bar{\Sigma}}^{2}+m_{\bar{Y}}^{2}-m_{Y}^{2}\right)\right] \\
\frac{d m_{\bar{\Sigma}}^{2}}{d t} & =\frac{1}{16 \pi^{2}}\left[\eta^{2}\left(2 m_{\bar{\Sigma}}^{2}+m_{\Sigma}^{2}+m_{X}^{2}\right)+4 \lambda_{Y}^{2}\left(\bar{m}_{\Sigma}^{2}+\bar{m}_{Y}^{2}\right)+\frac{g_{H}^{2}}{2}\left(-2 m_{\Sigma}^{2}+m_{Y}^{2}-m_{\bar{Y}}^{2}\right)\right] \\
\frac{d m_{Y}^{2}}{d t} & =\frac{1}{16 \pi^{2}}\left[\lambda_{Y}^{2}\left(4 m_{\Sigma}^{2}+6 m_{Y}^{2}\right)+\frac{g_{H}^{2}}{2}\left(-m_{\Sigma}^{2}+m_{\bar{\Sigma}}^{2}-\frac{1}{2} m_{\bar{Y}}^{2}\right)\right] \\
\frac{d m_{\bar{Y}}^{2}}{d t} & =\frac{1}{16 \pi^{2}}\left[\lambda_{\bar{Y}}^{2}\left(4 m_{\bar{\Sigma}}^{2}+6 m_{\bar{Y}}^{2}\right)+\frac{g_{H}^{2}}{2}\left(-m_{\bar{\Sigma}}^{2}+m_{\Sigma}^{2}-\frac{1}{2} m_{Y}^{2}\right)\right] \\
\frac{d m_{X}^{2}}{d t} & =\frac{1}{16 \pi^{2}}\left[\eta^{2}\left(m_{\Sigma}^{2}+m_{\bar{\Sigma}}^{2}+2 m_{X}^{2}\right)\right]
\end{aligned}
$$

Note that, without the interactions in eq. (A.1), the $m_{\Sigma, \bar{\Sigma}}^{2}$ equations can be rewritten in terms of $x \equiv m_{\Sigma}^{2}+m_{\bar{\Sigma}}^{2}$ so that both become $d x / d t \propto x$ whose solution never runs negative.

In figure 12, we plot contours of radiatively generated $\lambda^{\prime \prime}$ from eq. (2.12) in the $M_{\mathscr{D}}, \lambda_{Y}$ plane. For each contour, minimal gauge mediation defines the UV boundary condition $m_{\Sigma}=\frac{g_{H}^{2}}{16 \pi^{2}} \frac{F}{M_{*}}$ where $F$ saturates the bound in eq. (3.12). The allowed region assumes all couplings $\eta, \lambda_{Y, \bar{Y}}, g_{H}$ are all unity and we choose $\eta=0.1$ at the EW scale to generate a larger $v_{\Sigma}$ and satisfy the bounds on $\lambda^{\prime \prime}$ from eqs. (3.4) and (3.15). 

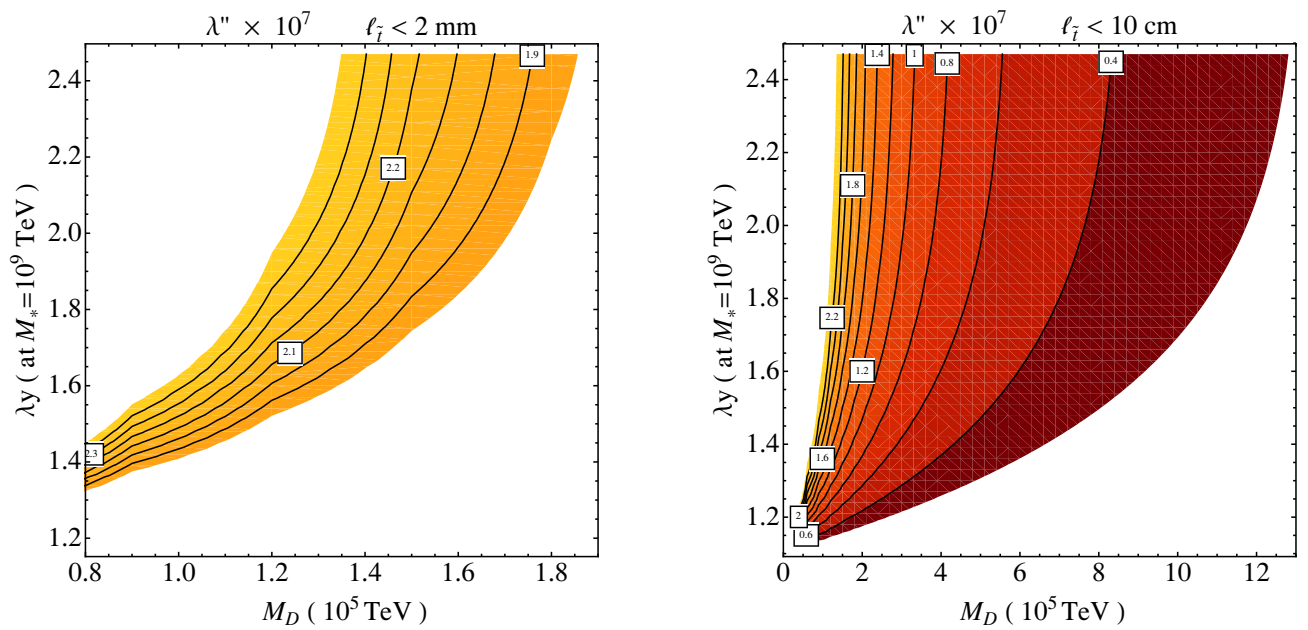

Figure 12. The allowed parameters space for $M_{\mathscr{D}}$ and $\lambda_{Y}$ with contours of $\lambda^{\prime \prime}$ derived from eq. (2.7). The white region is excluded by the dinucleon decay and displaced vertex bounds in section 3. For the left plot, we assume prompt stop decays with lengths $<2 \mathrm{~mm}$; for the right plot we assume displaced stop decays inside the tracker $(<10 \mathrm{~cm})$. The VEVs are computed after RG evolution with a UV boundary condition at the messenger scale, $M_{*}=10^{9} \mathrm{TeV}$, and IR boundary at the soft mass scale $m_{\mathcal{S}}=1 \mathrm{TeV}$. We also assume flavor universal couplings $|\kappa|$ and $\left|\kappa^{\prime}\right|$ and soft masses dictated by gauge mediation. Note that the range of $M_{\mathscr{D}}$ is of order the benchmark SUSY breaking scale $\sqrt{F} \sim 10^{5} \mathrm{TeV}$.

For this field content, radiative symmetry breaking requires $Y \bar{Y}$ to have larger soft masses than $\Sigma$ and $\bar{\Sigma}$ at the mediation scale, which is not realized in the minimal minimal gauge mediation; $\Sigma$ and $\bar{\Sigma}$ have larger gauge charges. However this can be accommodated if the $Y$ and $\bar{Y}$ carry additional gauge charges to give them larger soft masses at the mediation scale. Our example here assumes $m_{Y, \bar{Y}}\left(M_{*}\right)=2 m_{\Sigma, \bar{\Sigma}}\left(M_{*}\right)$ and suffices to demonstrate that radiative $R$-breaking is possible.

\section{B Color breaking?}

After $R$-breaking, up to order-one coefficients, the scalar potential derived from eq. (2.4) contains the terms

$$
V \supset\left|\frac{\widetilde{\bar{U}} \widetilde{\bar{D}}_{i} \widetilde{\bar{D}}_{j}}{M_{\mathscr{D}}}+v_{\Sigma}^{2}\right|^{2}+m_{\widetilde{u}}^{2}|\widetilde{\bar{U}}|^{2}+m_{\widetilde{d}_{i}}^{2}\left|\widetilde{\bar{D}}_{i}\right|^{2}+m_{\widetilde{d}_{j}}^{2}\left|\widetilde{\bar{D}}_{j}\right|^{2}
$$

which can break color if squark masses are too small. For simplicity, assuming identical squark soft masses and positive superpotential couplings, we can rewrite the potential in terms of dimensionless variables

$$
\hat{V} \equiv \frac{m_{\widetilde{d}_{i}}^{2} m_{\widetilde{d}_{j}}^{2}}{m_{\widetilde{u}}^{4} M_{\mathscr{D}}^{4}} V \supset\left|x y z+s^{2}\right|^{2}+\hat{m}^{2}\left(x^{2}+y^{2}+z^{2}\right)
$$

where

$$
x \equiv \frac{\langle\widetilde{\bar{U}}\rangle}{M_{\mathscr{D}}}, \quad y \equiv \frac{m_{\widetilde{d}_{i}}\left\langle\widetilde{\bar{D}}_{i}\right\rangle}{m_{\widetilde{u}} M_{\mathscr{D}}}, \quad z \equiv \frac{m_{\widetilde{d}_{j}}\left\langle\widetilde{\bar{D}}_{j}\right\rangle}{m_{\widetilde{u}} M_{\mathscr{D}}}, \quad s \equiv \frac{\sqrt{m_{\widetilde{d}_{i}} m_{\widetilde{d}_{j}}}}{m_{\widetilde{u}} M_{\mathscr{D}}} v_{\Sigma}, \quad \hat{m} \equiv \frac{m_{\widetilde{d}_{i}} m_{\widetilde{d}_{j}}}{m_{\widetilde{u}} M_{\mathscr{D}}} .
$$


At their extremal values, $x=y=z$, we demand

$$
\left(x^{3}+s^{2}\right)^{2}+3 \hat{m}^{2} x^{2} \geq s^{4},
$$

to avoid color breaking at the global minimum. This conditions implies, $s \lesssim \hat{m}^{3 / 4}$, so we need

$$
v_{\Sigma} \lesssim\left(\frac{m_{\widetilde{u}} m_{\widetilde{d}_{i}} m_{\widetilde{d}_{j}}}{M_{\mathscr{D}}^{3}}\right)^{1 / 4} M_{\mathscr{D}} .
$$

For the model's relevant parameter space, $m_{\widetilde{d}} \gtrsim 500 \mathrm{GeV}$ and $M \simeq 10^{5} \mathrm{TeV}$, this constraint becomes $v_{\Sigma}^{2} / M_{\mathscr{D}} \lesssim 10^{-4} \mathrm{TeV}$, which is an order of magnitude weaker than the dinucleon decay bound in eq. (3.6), so color remains unbroken for the viable parameter space we consider.

Open Access. This article is distributed under the terms of the Creative Commons Attribution License (CC-BY 4.0), which permits any use, distribution and reproduction in any medium, provided the original author(s) and source are credited.

\section{References}

[1] J.A. Evans and Y. Kats, LHC coverage of RPV MSSM with light stops, JHEP 04 (2013) 028 [arXiv: 1209.0764] [INSPIRE].

[2] C. Brust, A. Katz and R. Sundrum, SUSY stops at a bump, JHEP 08 (2012) 059 [arXiv:1206.2353] [INSPIRE].

[3] E. Nikolidakis and C. Smith, Minimal flavor violation, seesaw and R-parity, Phys. Rev. D 77 (2008) 015021 [arXiv:0710.3129] [INSPIRE].

[4] C. Csáki, Y. Grossman and B. Heidenreich, MFV SUSY: a natural theory for R-parity violation, Phys. Rev. D 85 (2012) 095009 [arXiv:1111.1239] [INSPIRE].

[5] G. Krnjaic and D. Stolarski, Gauging the way to MFV, JHEP 04 (2013) 064 [arXiv: 1212.4860] [INSPIRE].

[6] R. Franceschini and R. Mohapatra, New patterns of natural R-parity violation with supersymmetric gauged flavor, JHEP 04 (2013) 098 [arXiv:1301.3637] [INSPIRE].

[7] C. Csáki and B. Heidenreich, A complete model for R-parity violation, Phys. Rev. D 88 (2013) 055023 [arXiv: 1302.0004] [INSPIRE].

[8] J.T. Ruderman, T.R. Slatyer and N. Weiner, A collective breaking of R-parity, JHEP 09 (2013) 094 [arXiv:1207.5787] [INSPIRE].

[9] P. Fileviez Perez and M.B. Wise, Breaking local baryon and lepton number at the TeV scale, JHEP 08 (2011) 068 [arXiv: 1106.0343] [INSPIRE].

[10] D.S. Alves, P.J. Fox and N. Weiner, Supersymmetry with a sister Higgs, arXiv: 1207.5522 [INSPIRE].

[11] B. Bhattacherjee, J.L. Evans, M. Ibe, S. Matsumoto and T.T. Yanagida, Natural SUSY's last hope: R-parity violation via UDD operators, Phys. Rev. D 87 (2013) 115002 [arXiv: 1301.2336] [INSPIRE].

[12] B. Allanach, A. Dedes and H. Dreiner, $R$ parity violating minimal supergravity model, Phys. Rev. D 69 (2004) 115002 [Erratum ibid. D 72 (2005) 079902] [hep-ph/0309196] [INSPIRE]. 
[13] F.D. Steffen, Gravitino dark matter and cosmological constraints, JCAP 09 (2006) 001 [hep-ph/0605306] [INSPIRE].

[14] A. Florez, D. Restrepo, M. Velasquez and O. Zapata, Baryonic violation of $R$ parity from anomalous $\mathrm{U}(1)_{H}$, Phys. Rev. D 87 (2013) 095010 [arXiv: 1303.0278] [INSPIRE].

[15] J.R. Ellis, G. Gelmini, C. Jarlskog, G.G. Ross and J. Valle, Phenomenology of supersymmetry with broken R-parity, Phys. Lett. B 150 (1985) 142 [INSPIRE].

[16] J. Goity and M. Sher, Bounds on $\Delta B=1$ couplings in the supersymmetric standard model, Phys. Lett. B 346 (1995) 69 [Erratum ibid. B 385 (1996) 500] [hep-ph/9412208] [INSPIRE].

[17] B. Allanach, A. Dedes and H.K. Dreiner, Bounds on R-parity violating couplings at the weak scale and at the GUT scale, Phys. Rev. D 60 (1999) 075014 [hep-ph/9906209] [INSPIRE].

[18] S. Bar-Shalom and S. Roy, Effective R parity violation from supersymmetry breaking, Phys. Rev. D 69 (2004) 075004 [hep-ph/0304170] [INSPIRE].

[19] B. de Carlos and P. White, R-parity violation effects through soft supersymmetry breaking terms and the renormalization group, Phys. Rev. D 54 (1996) 3427 [hep-ph/9602381] [INSPIRE].

[20] I. Jack, D. Jones and A. Kord, $R$ parity violation and general soft supersymmetry breaking, Phys. Lett. B 588 (2004) 127 [hep-ph/0402045] [INSPIRE].

[21] R. Barbier et al., R-parity violating supersymmetry, Phys. Rept. 420 (2005) 1 [hep-ph/0406039] [INSPIRE].

[22] A. Kord and S. Hosseini, Two-loop R-parity violating renormalisation group equations for non-standard soft supersymmetry breaking in the context of the MSSM, arXiv:1111.0455 [INSPIRE].

[23] C. Frugiuele, T. Gregoire, P. Kumar and E. Ponton, ' $L=R$ ' $-\mathrm{U}(1)_{R}$ as the origin of leptonic 'RPV', JHEP 03 (2013) 156 [arXiv:1210.0541] [INSPIRE].

[24] A.E. Nelson and N. Seiberg, $R$ symmetry breaking versus supersymmetry breaking, Nucl. Phys. B 416 (1994) 46 [hep-ph/9309299] [INSPIRE].

[25] P.J. Fox, A.E. Nelson and N. Weiner, Dirac gaugino masses and supersoft supersymmetry breaking, JHEP 08 (2002) 035 [hep-ph/0206096] [INSPIRE].

[26] G.D. Kribs, E. Poppitz and N. Weiner, Flavor in supersymmetry with an extended R-symmetry, Phys. Rev. D 78 (2008) 055010 [arXiv:0712.2039] [INSPIRE].

[27] A. Aranda, C. Bonilla, S. Morisi, E. Peinado and J. Valle, Dirac neutrinos from flavor symmetry, arXiv:1307.3553 [INSPIRE].

[28] G.G. Raffelt, Astrophysical methods to constrain axions and other novel particle phenomena, Phys. Rept. 198 (1990) 1 [INSPIRE].

[29] J. Bagger, E. Poppitz and L. Randall, The R axion from dynamical supersymmetry breaking, Nucl. Phys. B 426 (1994) 3 [hep-ph/9405345] [InSPIRE].

[30] ATLAS collaboration, Search for displaced vertices arising from decays of new heavy particles in $7 \mathrm{TeV}$ pp collisions at ATLAS, Phys. Lett. B 707 (2012) 478 [arXiv:1109.2242] [INSPIRE].

[31] ATLAS collaboration, Search for pair production of massive particles decaying into three quarks with the ATLAS detector in $\sqrt{s}=7$ TeV pp collisions at the LHC, JHEP 12 (2012) 086 [arXiv:1210.4813] [INSPIRE]. 
[32] D. Curtin, R. Essig and B. Shuve, Boosted multijet resonances and new color-flow variables, Phys. Rev. D 88 (2013) 034019 [arXiv: 1210.5523] [InSPIRE].

[33] T. Cohen, E. Izaguirre, M. Lisanti and H.K. Lou, Jet substructure by accident, JHEP 03 (2013) 161 [arXiv:1212.1456] [INSPIRE].

[34] M. Asano, K. Rolbiecki and K. Sakurai, Can R-parity violation hide vanilla supersymmetry at the LHC?, JHEP 01 (2013) 128 [arXiv:1209.5778] [INSPIRE].

[35] Z. Han, A. Katz, M. Son and B. Tweedie, Boosting searches for natural SUSY with RPV via gluino cascades, Phys. Rev. D 87 (2013) 075003 [arXiv:1211.4025] [inSPIRE].

[36] J. Berger, M. Perelstein, M. Saelim and P. Tanedo, The same-sign dilepton signature of RPV/MFV SUSY, JHEP 04 (2013) 077 [arXiv: 1302.2146] [INSPIRE].

[37] M.D. Litos, A search for dinucleon decay into kaons using the Super-Kamiokande water Cherenkov detector, Ph.D. thesis, Boston University, Boston U.S.A. (2010).

[38] Super-Kamiokande collaboration, K. Abe et al., The search for $n-\bar{n}$ oscillation in Super-Kamiokande I, arXiv:1109.4227 [INSPIRE].

[39] Particle Data Group collaboration, J. Beringer et al., Review of particle physics, Phys. Rev. D 86 (2012) 010001 [inSPIRE].

[40] M. Asano, T. Ito, S. Matsumoto and T. Moroi, Exploring supersymmetric model with very light gravitino at the LHC, JHEP 03 (2012) 011 [arXiv:1111.3725] [INSPIRE].

[41] P.W. Graham, D.E. Kaplan, S. Rajendran and P. Saraswat, Displaced supersymmetry, JHEP 07 (2012) 149 [arXiv: 1204.6038] [INSPIRE].

[42] A. Masiero and J. Valle, A model for spontaneous $R$ parity breaking, Phys. Lett. B 251 (1990) 273 [INSPIRE].

[43] M. Hirsch and J. Valle, Supersymmetric origin of neutrino mass, New J. Phys. 6 (2004) 76 [hep-ph/0405015] [INSPIRE].

[44] D. Restrepo, M. Taoso, J. Valle and O. Zapata, Gravitino dark matter and neutrino masses with bilinear R-parity violation, Phys. Rev. D 85 (2012) 023523 [arXiv:1109.0512] [INSPIRE].

[45] A. Arvanitaki, M. Baryakhtar, X. Huang, K. van Tilburg and G. Villadoro, The last vestiges of naturalness, JHEP 03 (2014) 022 [arXiv: 1309.3568] [INSPIRE].

[46] J.R. Ellis, J. Gunion, H.E. Haber, L. Roszkowski and F. Zwirner, Higgs bosons in a nonminimal supersymmetric model, Phys. Rev. D 39 (1989) 844 [INSPIRE].

[47] Y. Okada, M. Yamaguchi and T. Yanagida, Upper bound of the lightest Higgs boson mass in the minimal supersymmetric standard model, Prog. Theor. Phys. 85 (1991) 1 [INSPIRE].

[48] H.E. Haber and R. Hempfling, Can the mass of the lightest Higgs boson of the minimal supersymmetric model be larger than $m(Z)$ ?, Phys. Rev. Lett. 66 (1991) 1815 [INSPIRE].

[49] J.R. Ellis, G. Ridolfi and F. Zwirner, On radiative corrections to supersymmetric Higgs boson masses and their implications for LEP searches, Phys. Lett. B 262 (1991) 477 [INSPIRE].

[50] P. Batra, A. Delgado, D.E. Kaplan and T.M. Tait, The Higgs mass bound in gauge extensions of the minimal supersymmetric standard model, JHEP 02 (2004) 043 [hep-ph/0309149] [INSPIRE]. 
[51] R. Harnik, G.D. Kribs, D.T. Larson and H. Murayama, The minimal supersymmetric fat Higgs model, Phys. Rev. D 70 (2004) 015002 [hep-ph/0311349] [INSPIRE].

[52] S. Chang, C. Kilic and R. Mahbubani, The new fat Higgs: slimmer and more attractive, Phys. Rev. D 71 (2005) 015003 [hep-ph/0405267] [InSPIRE].

[53] A. Maloney, A. Pierce and J.G. Wacker, D-terms, unification and the Higgs mass, JHEP 06 (2006) 034 [hep-ph/0409127] [INSPIRE].

[54] J. Galloway, M.A. Luty, Y. Tsai and Y. Zhao, Induced electroweak symmetry breaking and supersymmetric naturalness, arXiv:1306.6354 [INSPIRE].

[55] J.L. Feng, Z. Surujon and H.-B. Yu, Confluence of constraints in gauge mediation: the 125 GeV Higgs boson and goldilocks cosmology, Phys. Rev. D 86 (2012) 035003 [arXiv: 1205.6480] [INSPIRE].

[56] N. Craig, The state of supersymmetry after Run I of the LHC, arXiv:1309.0528 [INSPIRE]. 